\title{
Mutational Analysis and Modeling Reveal Functionally Critical Residues in Transmembrane Segments 1 and 3 of the UapA Transporter
}

\section{Sotiris Amillis ${ }^{1}$, Vasiliki Kosti ${ }^{1}$, Areti Pantazopoulou ${ }^{1}$, Emmanuel Mikros ${ }^{2}$ and George Diallinas ${ }^{1 *}$}

\author{
${ }^{1}$ Department of Botany, Faculty of Biology, University of Athens, Panepistimioupolis, Athens 15781, Greece \\ ${ }^{2}$ School of Pharmacy, University of Athens, Panepistimioupolis, Athens 15771, Greece
}

Received 15 April 2011; received in revised form 9 June 2011; accepted 14 June 2011 Available online 29 June 2011

\section{Edited by I. B. Holland}

Keywords:

Aspergillus nidulans; nucleobase ascorbate transporter (NAT) family; suppressors; uric acid/xanthine; topological modeling
Earlier, we identified mutations in the first transmembrane segment (TMS1) of UapA, a uric acid-xanthine transporter in Aspergillus nidulans, that affect its turnover and subcellular localization. Here, we use one of these mutations (H86D) and a novel mutation (I74D) as well as genetic suppressors of them, to show that TMS1 is a key domain for proper folding, trafficking and turnover. Kinetic analysis of mutants further revealed that partial misfolding and deficient trafficking of UapA does not affect its affinity for xanthine transport, but reduces that of uric acid and confers a degree of promiscuity towards the binding of other purines. This result strengthens the idea that subtle interactions among domains not directly involved in substrate binding refine the selectivity of UapA. Characterization of second-site suppressors of H86D revealed a genetic interaction of TMS1 with TMS3, the latter segment shown for the first time to be important for UapA function. Systematic mutational analysis of polar and conserved residues in TMS3 showed that Ser154 is crucial for UapA transport activity. Our results are in agreement with a topological model of UapA built on the recently published structure of UraA, a bacterial homolog of UapA.

(c) 2011 Elsevier Ltd. All rights reserved.

\section{Introduction}

The family of nucleobase-ascorbate transporters (NATs), also called the nucleobase-cation symporter-2 (NCS2) family†, consists of hundreds of

${ }^{*}$ Corresponding author. E-mail address:

diallina@biol.uoa.gr.

Present address: A. Pantazopoulou, Department of Molecular and Cellular Medicine, Centro de Investigaciones Biológicas CSIC, Ramiro de Maeztu 9, Madrid 28040, Spain.

Abbreviations used: NAT, nucleobase-ascorbate transporter; TMS, transmembrane segment; GFP, green fluorescent protein.

† http: / / www.tcdb.org/2.A.40 members present in nearly all organisms, a prominent exception being Saccharomyces cerevisiae and several protozoa. At present, the function and specificity of nearly 20 NAT proteins are known. ${ }^{1-3}$ These proteins come from bacteria, fungi, plants and mammals. All non-mammalian homologs of known function are specific for nucleobases; namely, xanthine, uric acid or uracil. ${ }^{1,2}$ The mammalian NATs transport either L-ascorbic acid (SVCT1 and SVCT2 proteins in several mammals, including humans ${ }^{4,5}$ or nucleobases; namely, uracil, xanthine or hypoxanthine (in the rat homolog SNBT1 ${ }^{6)}$. The bacterial, fungal and plant NATs are high-affinity $\mathrm{H}^{+}$symporters, whereas the mammalian homologs use $\mathrm{Na}^{+}$ for L-ascorbate or nucleobase symport. ${ }^{1-3,6}$ All NATs are predicted to contain 12-14 putative transmembrane segments (TMS) and cytoplasmically located $\mathrm{N}$ - and C-tails, but there was no direct structural 
data for any member of this family until very recently (see below).

The UapA transporter of the filamentous fungus Aspergillus nidulans ${ }^{7,8}$ is the prototype of the NAT family and one of the most extensively studied carriers concerned with regulation of transcription ${ }^{9,10}$ and endocytosis via ubiquitination, ${ }^{11}$ but mostly with respect to structure-function relationships. ${ }^{1,2,12}$ Through many rounds of alternating rationale and random mutational approaches and construction of chimeric molecules, combined with physiological tests, detailed analysis of transport kinetics and the use green fluorescent protein (GFP)-tagged versions of mutated UapA molecules, we have proposed models to explain how UapA functions and selects its substrates, despite the fact that no direct structural study is available. ${ }^{13-19}$

In particular, it is known that UapA is a highaffinity, high-capacity symporter of $\mathrm{H}^{+}$and xanthine or uric acid and that several analogs of these purines, especially those that do not have modifications in positions N1-H and N9 or = O8 of the purine ring, can also act as substrates or ligands of UapA, albeit with lower affinity. Four fully conserved amino acid residues (Q85, E356, D388 and N409) have been found to be irreplaceable for function, whereas eight more partially conserved residues (N71, Q113, Q408, G411, T417, R481, T526 and F528) are crucial for determining the transport kinetics and substrate specificity of UapA. According to standard topological algorithms, all functionally important residues in UapA map in four distinct regions; TMS1 and its flanking segments, the amphipathic segment including TMS8-TMS9, the TMS10-TMS11 cytoplasmic loop and TMS12. Strong genetic and biochemical evidence has shown that the amphipathic TMS8-TMS9 region, which includes most of the functionally important residues, hosts the basic elements of the substrate-binding site. ${ }^{16-19}$ One residue in this region, Q408, has been shown to be involved in direct contacts with either N9 or $=$ O8 of xanthine or uric acid, respectively. The other three functional regions (TMS1, TMS10TMS11 cytoplasmic loop and TMS12) appear to interact dynamically with each other and with the TMS8-TMS9 substrate-binding domain, acting as gating elements or selectivity filters that determine the substrate affinity and specificity of UapA. ${ }^{19}$ Most of the above conclusions concerning UapA, especially those concerning the TMS8-TMS9 domain and TMS12 region, are supported by reverse genetics and Cys-scanning mutational analysis of the XanQ xanthine transporter in Escherichia coli, which is the most extensively studied bacterial member of the NAT family. ${ }^{20-24}$

Interestingly, the great majority of functional mutations of UapA do not affect the folding or turnover of UapA, as judged by the normal localization of the relevant mutant forms of GFP- tagged transporter in the plasma membrane. In fact, the only mutations affecting UapA localization and turnover are a handful of substitutions of residues in TMS $^{25}$ or deletions/insertions of one or two residues in the loop linking TMS10 with TMS11. ${ }^{26}$ In this work, we used previously characterized and novel TMS1 mutations that result in ER-retention and/or increased turnover of UapA, as well as genetic suppressors of these mutations, to show that TMS1 and a TMS1-TMS3 interaction are crucial for proper folding, trafficking and turnover of UapA. Subsequently, using a systematic mutational analysis, we show that Ser154 within TMS3 is crucial for UapA transport activity. Our results are evaluated in relation to a three-dimensional topological model of UapA built on the recently published structure of UraA, a bacterial NAT homolog.

\section{Results}

\section{TMS1 mutations affect UapA folding, trafficking and turnover}

H86 mutations (H86A, H86D and H86K) isolated earlier lead to increased turnover of UapA through sorting and degradation in the vacuoles at $25^{\circ} \mathrm{C} .{ }^{25}$ H86D also leads to partial retention of UapA in the ER membrane. Mutation I74D constructed here is just upstream from the putative TMS1 and leads to non-conditional, complete ER-retention of UapA. The subcellular localization of GFP-tagged versions of UapA-I74D, UapA-H86A and UapA-H86D is shown in Figure 1a. The relevant mutants have significantly reduced (H86A and H86D) or abolished (I74D) UapA-mediated radiolabeled $\left[{ }^{3} \mathrm{H}\right]$ xanthine uptake, which is reflected in the inability to grow with uric acid (Fig. 1b) or xanthine (not shown) as sole nitrogen source, given other purine transporters besides UapA are knocked-out (strain details are given in Materials and Methods). Deficient localization or/and increased turnover of transporters is expected to be associated with defective folding. A defect in folding was suggested by the cryosensitive nature of H86 mutations. We showed directly that this is the case for the UapAH86D mutant, where ER retention is indeed associated with evidence for an unfolded protein response. ${ }^{27}$ As expected, the UapA-H86A mutant, which is not retained in the ER, similar to wild type, did not show any evidence for an unfolded protein response (UPR: Fig. 1c). Interestingly, however, in the case of H86 mutations, where a fraction of UapA reaches the plasma membrane and thus allows direct measurements of transport rates and $K_{\mathrm{m}}$ values for xanthine, uric acid or other purines, folding defects have been shown not to affect the activity of UapA significantly. ${ }^{25}$ 
(a)

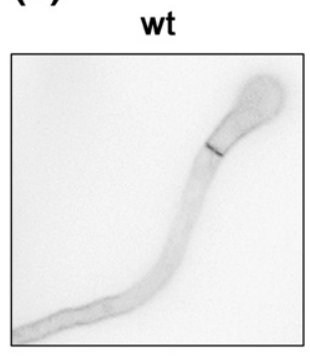

H86A-GFP

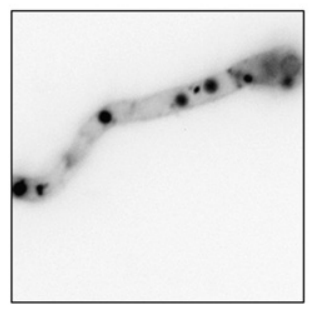

174D-GFP

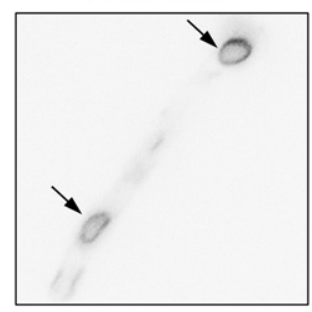

H86D-GFP

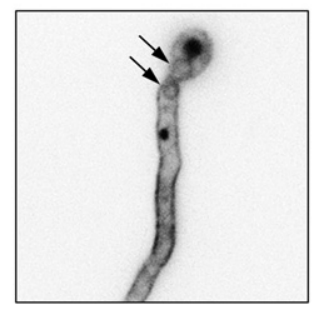

(b)

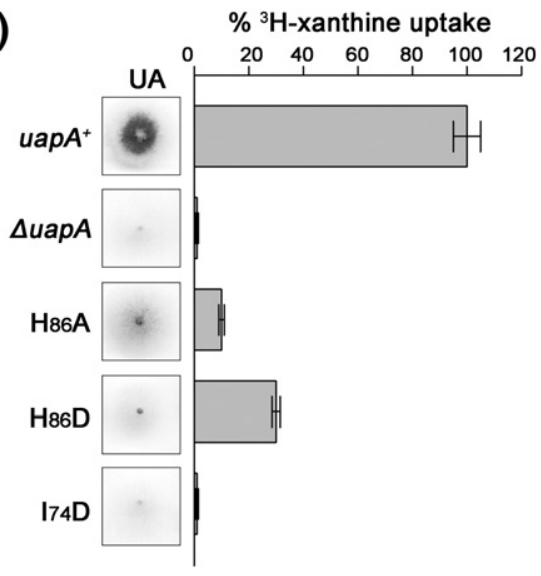

(c)

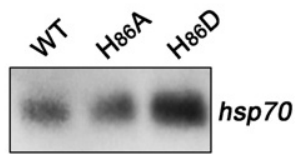

Fig. 1. Functional characterization of TMS1 mutations. (a) Epifluorescent microscopic analysis of GFP-tagged wild type (wt) and mutant alleles (H86A, H86D and I74D) of UapA. UapA-GFP labels primarily the plasma membrane in wt and H86D, but distinct cytoplasmically labeled structures appear in all mutants. Arrows in H86D and I74D depict perinuclear ER rings (membranes). UapA-GFP in H86A labels the plasma membrane but mostly cytoplasmic compact structures, which correspond to vacuoles. (b) Growth with $0.5 \mathrm{mM}$ uric acid (UA) as sole nitrogen source and UapA-mediated radiolabeled ${ }^{3} \mathrm{H}$-xanthine initial uptake rates (\%) in control strains (uap $A^{+}$and $\Delta$ uapA) and TMS1 mutants. Values represent averages of three independent experiments with standard deviation $<20 \%$. (c) Evidence for unfolded protein response in the mutants expressing UapA-H86D. hsp70 (ANID_02062.1) transcript study-state levels detected by northern blot analysis of total RNA (10 $\mu \mathrm{g}$ in all lanes). A five-fold increase was estimated in H86D compared to wt or H86A.

\section{Genetic suppression of TMS1 mutations}

In order to further understand how missense mutations in TMS1 affect UapA turnover and subcellular trafficking, we selected genetic revertants of mutations H86D and I74D by standard UV mutagenesis. The relevant mutants expressing UapA-H86D or UapA-I74D in an appropriate genetic background cannot grow with uric acid as sole nitrogen source at $25{ }^{\circ} \mathrm{C}$ (Materials and Methods). We obtained and characterized several revertants of both mutants as colonies able to grow with uric acid as sole nitrogen source. In both cases, all revertants were found to be due to genetic suppressor mutations located in UapA. The identity and location of these suppressors is summarized in Table 1 . In both cases, mutagenesis was apparently close to saturation because several suppressors were obtained twice or

Table 1. Identity and location of I74D and H86D suppressors

\begin{tabular}{lccr}
\hline Amino acid substitution & Topology in UapA & Nucleotide change & Numbers obtained \\
\hline I74D & N-terminal/TMS1 & ATT $\rightarrow$ GAT & - \\
A. Suppressors of I74D & N-terminal/TMS1 & GAT $\rightarrow$ GGT & 2 \\
I74G & N-terminal/TMS1 & GAT $\rightarrow$ GTT & 2 \\
I74V & N-terminal/TMS1 & GAT $\rightarrow$ GCT & 2 \\
I74A & N-terminal/TMS1 & GAT $\rightarrow$ AAT & 1 \\
I74N & TMS1 & CAT $\rightarrow$ GAT & - \\
H86D & & GAT $\rightarrow$ GTT & 2 \\
B. First-site suppressors of H86D & TMS1 & GAT $\rightarrow$ AAT & 5 \\
H86V & TMS1 & ATG $\rightarrow$ ATA & 1 \\
C. Second-site suppressors of H86D & & ATG $\rightarrow$ GTG & 1 \\
M151I & TMS3 & TMS3 & \\
M151V & &
\end{tabular}

The nucleobase substitutions of the wild type uapA codon are shown in bold. Suppressors are indicated as substitutions of the original amino acid residue (Ile74 or His86). 
(a)

(b) Uptake of ${ }^{3} \mathrm{H}$-xanthine at $25^{\circ} \mathrm{C}$
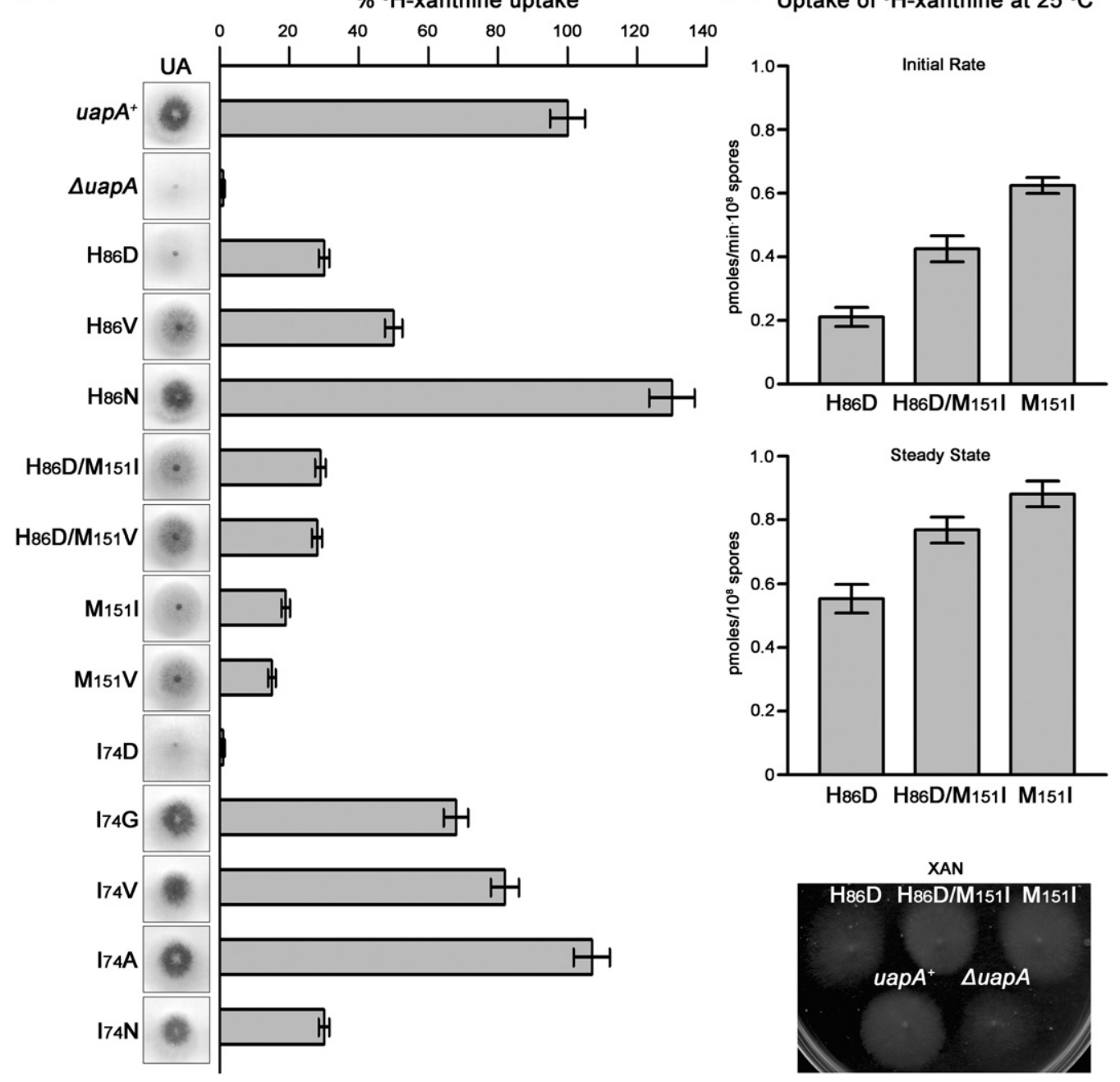

Fig. 2. (a) Comparative growth tests at $25{ }^{\circ} \mathrm{C}$ with $0.5 \mathrm{mM}$ uric acid (UA) as sole nitrogen source and UapA-mediated radiolabelled ${ }^{3} \mathrm{H}$-xanthine uptake rates (\%) in control strains (uap $A^{+}$and $\Delta$ uapA), TMS1 mutations and their suppressors at $37^{\circ} \mathrm{C}$. (b) Comparative growth tests $25^{\circ} \mathrm{C}$ with $1 \mathrm{mM}$ xanthine (XAN) as sole nitrogen source at and UapA-mediated radiolabeled ${ }^{3} \mathrm{H}$-xanthine initial uptake rates or steady-state uptake levels in the H86D mutation and its suppressor. Values are averages of three independent experiments with standard deviation $<20 \%$.

more, or concerned the same codon. All I74D suppressors corresponded to changes in the originally mutated codon (first site-suppressors), whereas H86D revertants were due to both first site-suppressors and mutations replacing Met151 in TMS3 by Val or Ile (second site-suppressors H86D/M151V and H86D/M151I). At this point, we constructed isogenic strains expressing UapA-M151V and UapA-M151I alleles by themselves; i.e. in the absence of the original H86D mutation (Materials and Methods).

\section{Functional characterization, expression and subcellular localization of UapA in suppressors}

Figure 2 shows comparative growth tests and xanthine initial uptake rates of all suppressors, original mutants and control strains. It was apparent that with respect to Ile74, mutations introducing hydrophobic amino acids (Ala, Gly and Val) restored at least $70 \%$ of the apparent UapA initial transport rate, whereas introduction of Asn did so only partially $(30 \%)$. Among H86D suppressors, only the presence of Asn in the originally mutated codon fully restored UapA transport rate $(\sim 130 \%)$, whereas the presence of Val in that codon partially re-established the transport rate of UapA (54\%). Surprisingly, second site-suppressors M151V or M151I did not lead to any change in xanthine initial uptake rates $(28-29 \%)$ in relation to the original mutated strain expressing H86D (30\%). This suggested that substitutions in M151 might have restored, at least to some degree, the turnover rather than the function of plasma 
Table 2. Substrate specificity profile of mutants and suppressors

\begin{tabular}{lccc}
\hline$K_{\mathrm{m} / \mathrm{i}}(\mu \mathrm{M})$ & & & \\
\hline Allele & Xanthine & Uric acid & Hypoxanthine \\
\hline Wt & 7 & 8 & n.i. \\
I74D & n.d. & n.d. & n.d. \\
I74G & 8 & 35 & $\sim 1000$ \\
I74V & 28 & 102 & n.i. \\
I74A & 7 & 195 & $\sim 1000$ \\
I74N & 5 & 8 & 280 \\
H86A & 7 & 23 & n.i. \\
H86D & 4 & 15 & n.i. \\
H86V & 19 & 29 & n.i. \\
H86N & 4 & 28 & n.i. \\
H86D/M151I & 4 & 18 & n.i. \\
H86D/M151V & 14 & 17 & n.i. \\
M151I & 25 & 34 & n.i. \\
M151V & 15 & 107 & n.i. \\
\hline K & & &
\end{tabular}

$K_{\mathrm{m} / \mathrm{i}}$ values were determined as described in Materials and Methods. n.d., not determined. n.i., no inhibition $(90-100 \%$ uptake). The tilde $(\sim)$ identifies values $\geq 1000 \mu \mathrm{M}$ that were estimated. The results are averages of at least three independent experiments with three replicates for each concentration and the standard deviation was $<20 \%$.

membrane-embedded UapA-H86D molecules. Relatively low transport capacities were exhibited by the single mutations M151V and M151I (16-22\%).

We investigated in more detail the nature of the suppressors isolated by (i) measuring $K_{\mathrm{m}}$ values for substrates, (ii) establishing an extended substrate specificity profile through competition assays $\left(K_{\mathrm{i}}\right.$ values) and (iii) following the turnover and trafficking of the mutated UapA versions.

Table 2 shows that none of the I74D or H86D suppressors affect the affinity for xanthine significantly. For example, even in the case of the maximal (approx. four-fold) increases in $K_{\mathrm{m}}$, as is the case in I74V or $\mathrm{M} 151 \mathrm{~V}$, this change corresponds to a reduction of less than $3 \mathrm{~kJ} / \mathrm{mol}$ in the $\Delta G^{0}$ values governing transportsubstrate interactions. ${ }^{17}$ Interestingly, however, for some I74D suppressors, significantly higher affinity changes were observed specifically for uric acid, the other physiological substrate of UapA. This is highlighted in I74V and I74A mutants, which showed 13-fold and 24-fold increases in the $K_{\mathrm{i}}$ values for uric acid, respectively. We estimated $K_{\mathrm{i}}$ values for hypoxanthine, a purine not recognized by wild type UapA. Three Ile74 mutants showed low binding affinity for hypoxanthine with values of $280 \mu \mathrm{M}$ for I74N and $\sim 1 \mathrm{mM}$ for I74A and I74G (Table 2). These mutants could also recognize adenine, guanine or uracil with very low $(1 \sim 2 \mathrm{mM}$ ) affinity (data not shown). Growth tests showed that none of the Ile74 mutants could grow on purines other than xanthine or uric acid, which indicates that even in the case of $\mathrm{I} 74 \mathrm{~N}, \mathrm{I} 74 \mathrm{~A}$ and $\mathrm{I} 74 \mathrm{G}$, purines that are non-physiological substrates of UapA are transported very inefficiently or not at all (not shown).
Figure 3a shows a western blot analysis of UapAGFP in control strains and mutants H86A, H86D, H86D/M151I, I74D, I74N and I74G. Quantification of the steady-state levels of the UapA-GFP protein was done using the protein levels of the constitutively expressed actin gene as an internal control. H86A is the only mutant with a significantly increased turnover compared to wild type $(43 \%$ of the wild type UapA-GFP). UapA-GFP levels were also moderately decreased ( $69 \%$ of the wild type) in suppressor H86D/M151I. However, the presence of intact UapAGFP does not distinguish whether the tagged transporter is in the plasma membrane or in any other internal membrane. Thus, to further analyze the effect of the different suppressor mutations isolated, we examined the subcellular localization of UapAGFP in the relevant mutant strains. Figure $3 b$ (lower panel) shows that suppressors of I74D introducing small hydrophobic residues (Ala, Gly or Val) fully restored UapA-GFP localization. Introducing a polar residue at Ile74 (I74N) suppressed the ER-retention defect observed with I74D, but led to significantly increased vacuolar sorting of UapA molecules. Firstsite suppressors of H86D (H86N and H86V) also fully restored UapA-GFP localization (not shown). Finally, the second site-suppressor mutation M151I only partially restored the localization defect (e.g. ERretention) of UapA molecules carrying H86D (Fig. 3b, upper right panel). These results were in line with those from growth tests, western analysis and kinetic measurements.

\section{Mutational and functional analysis of polar and conserved residues of TMS3}

Second site-suppressors of H86D concerned substitutions of Met151, a residue in the middle of the putative TMS3. This segment was predicted, using various algorithms to form an amphipathic $\alpha$-helix. However, a three-dimensional topological model of UapA (see below) built on the recently published structure of the UraA (uracil) permease of Escherichia coli $^{28}$ shows that TMS3 includes a short $\beta$-strand that includes Met151 (residues 150-153). Six partially conserved Ser residues (positions 145, 149, 154, 156,159 and 162) flank the $\beta$-strand segment and should create a polar interface along TMS3 (Fig. 4a). We substituted all Ser residues of TMS3 with Ala residues (Materials and Methods) and analyzed the relevant mutants. We also mutated the only wellconserved residue of TMS3; Phe155, which is present in all fungal and bacterial NAT homologs (Fig. 4a). Growth tests (Fig. 4b) showed that only two residues were functionally important; S154A

$\$$ thtp:/ / www.cbs.dtu.dk/services / TMHMM2, http://bp.nuap.nagoyau.ac.jp/sosui/, http://www.ch. embnet.org/software/TMPRED 


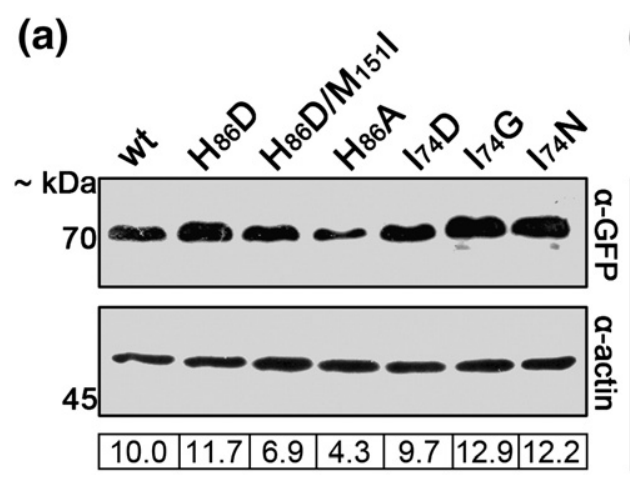

(b)
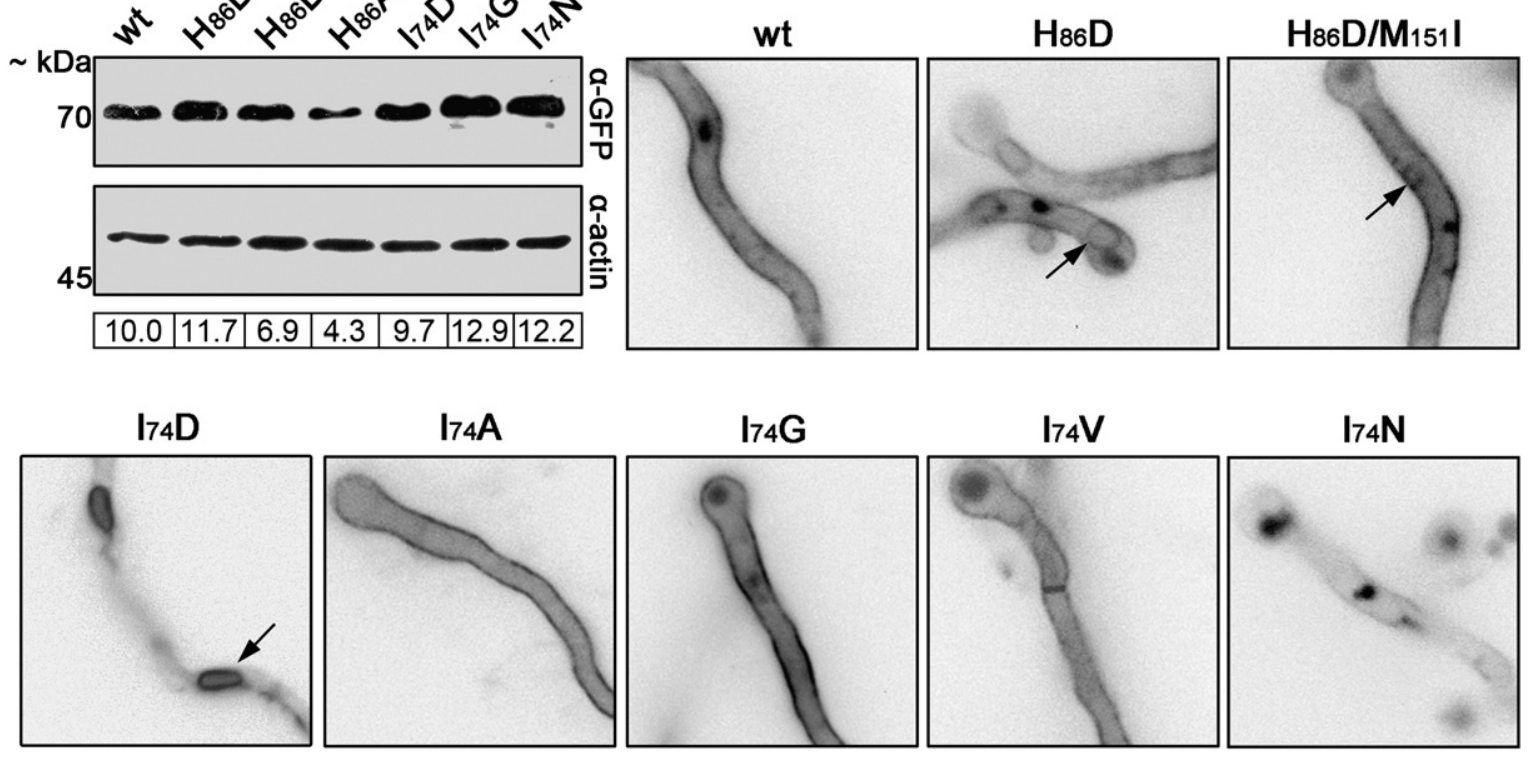

Fig. 3. (a) Western blot analysis of UapA-GFP in control strains and TMS1 mutants and suppressors. Equal amounts of total protein extracts $(50 \mu \mathrm{g})$ were loaded in all cases, transferred onto PVDF membranes and hybridized with oligoclonal anti-GFP and anti-actin antibodies. Relative amounts of UapA-GFP were estimated by normalizing with the wt UapAGFP/actin ratio (value set to 10.0) using ImageJ software. (b) Epifluorescent microscopic analysis of GFP-tagged wt and mutant alleles. Arrows indicate perinuclear ER membranes.

led to significantly reduced growth at $37{ }^{\circ} \mathrm{C}$ and S162A led to reduced growth at $25^{\circ} \mathrm{C}$, both with uric acid as the sole nitrogen source. A similar growth profile was obtained with xanthine as the sole nitrogen source (data not shown). None of the TMS3 mutants analyzed could grow with any other purine as sole nitrogen source (data not shown). Subcellular localization analysis (Fig. 4d) showed that none of the TMS3 mutations affected the localization of UapA-GFP in the plasma membrane. All mutants showed detectable UapA-mediated transport (34-127\% of wild type values), with S154A and S162A exhibiting the lowest transport rates ( $34 \%$ and $45 \%$, respectively) (Fig. 4c).

On the basis of two observations, we studied the role of Ser154 in UapA function in more detail. First, Ser154 in UapA corresponds to Asn93 in the E. coli xanthine permease XanQ. An Asn at this position has been shown to be crucial for substrate specificity in bacterial NATs (Discussion). ${ }^{22}$ Second, Ser154 has a crucial position with respect to the putative substrate-binding site in the UapA structural model constructed in this study (see below). We constructed mutation S154N and showed that it did not affect UapA-GFP localization in the plasma membrane (Fig. $4 \mathrm{~d}$ ) but had a dramatic effect on the apparent UapA transport capacity. This is shown by the lack of growth at $37^{\circ} \mathrm{C}$ and at $25^{\circ} \mathrm{C}$ with uric acid as sole nitrogen source (Fig. 4b) and the low $(20 \%)$ UapA-mediated transport rate of xanthine (Fig. 4c) in the corresponding mutant. When the S154A and S154N alleles were expressed in highcopy plasmid transformants, partial restoration of the ability to transport xanthine or uric acid was established, mostly in the case of S154A. In principle, restoration of a defect in the apparent transport capacity of UapA by high-copy expression implies that the relevant mutations affect either the intrinsic transport activity and/or the turnover of the transporter. However, in the case of Ser154

Fig. 4. Functional analysis of TMS3 mutants. (a) Multiple alignments of TMS1 and TMS3 of selected NAT sequences. Conserved and partially conserved residues are highlighted in black and grey boxes, respectively. In TMS1, the position of mutations I74D and H86D is indicated. In TMS3, the positions of all mutants studied here are indicated by arrows. Secondary structures as predicted by modeling of UapA topology on the UraA crystal structure are indicated under the alignments. Cylinders denote $\alpha$-helices. A filled flesh denotes a segment-forming $\beta$-strand. (b) Comparative growth tests of TMS3 mutants with $5 \mathrm{mM}$ urea (U) or $0.5 \mathrm{mM}$ uric acid (UA) as sole nitrogen sources at $25^{\circ} \mathrm{C}$ or $37^{\circ} \mathrm{C}$. (c) Comparative UapA-mediated radiolabeled ${ }^{3} \mathrm{H}$-xanthine uptake rates (\%) in control strains (uap $A^{+}$and $\Delta$ uapA) and TMS3 mutants in the absence or in the presence of non-radiolabelled substrate (xanthine $1 \mathrm{mM}$ ). (d) Epifluorescence microscopy of the subcellular localization of UapA-GFP in a control strain and TMS3 mutants. 

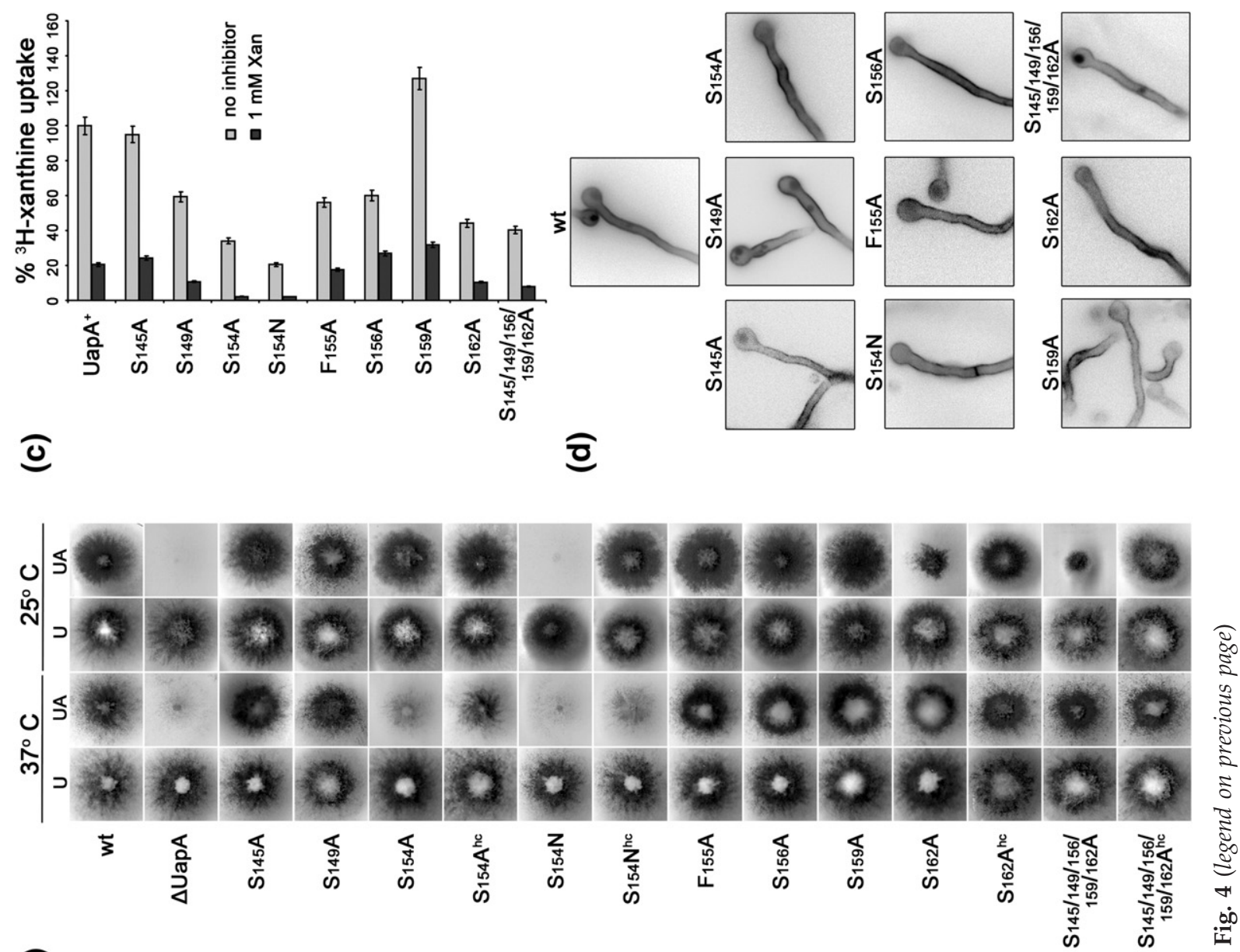

$\widehat{3}$
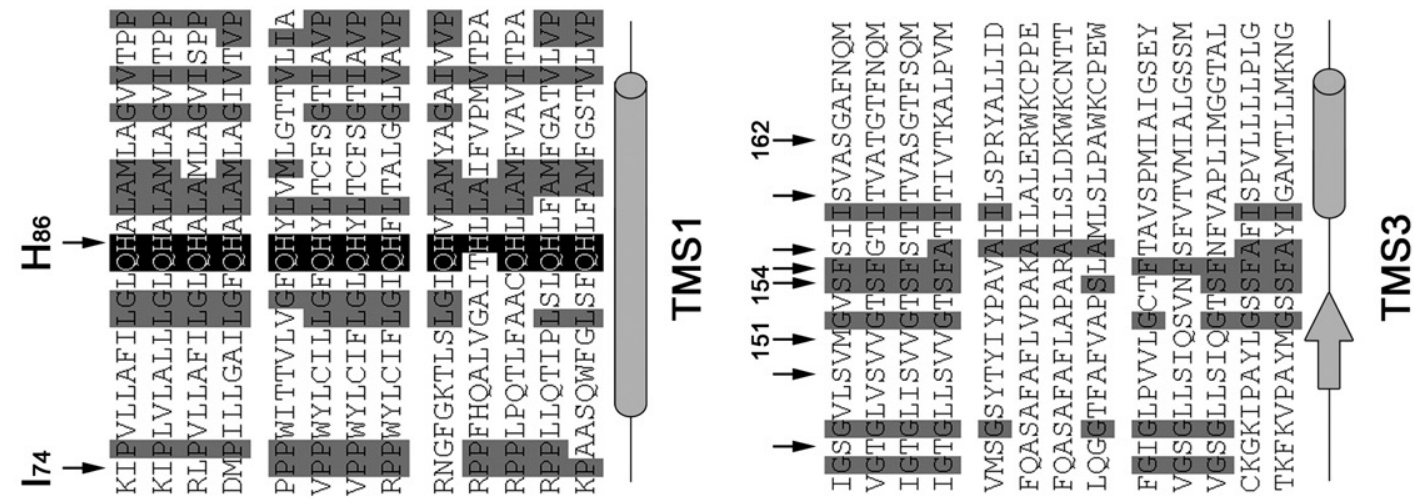

ฮ

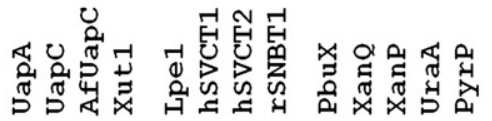


Table 3. Substrate specificity profile of S154 mutations

\begin{tabular}{lccc}
\hline$K_{\mathrm{m} / \mathrm{i}}(\mu \mathrm{M})$ & & & \\
\hline Substrate/ligand & $\mathrm{UapA}^{+}$ & $\mathrm{S} 154 \mathrm{~N}$ & $\mathrm{~S} 154 \mathrm{~A}$ \\
\hline Xanthine & 8 & 1.4 & 7.6 \\
Uric acid & 7 & 10 & 53 \\
Adenine & $\mathrm{n} . \mathrm{i}$ & $\mathrm{n} . \mathrm{i}$ & $>1000$ \\
Hypoxanthine & $\mathrm{n} . \mathrm{i}$ & $\mathrm{n} . \mathrm{i}$ & $>1000$ \\
Guanine & $\mathrm{n} . \mathrm{i}$. & $\mathrm{n} . \mathrm{i}$ & $>1000$ \\
Uracil & n.i. & 523 & $>1000$ \\
2-Thio-xanthine & 63 & 35 & 48 \\
3-Methyl-xanthine & 28 & 47 & 62 \\
6-Thio-xanthine & 350 & 326 & 553 \\
7-Methyl-xanthine & n.i. & 1000 & $\mathrm{n} . \mathrm{i}$ \\
8-Methyl-xanthine & 100 & 194 & 188 \\
9-mEthyl-xanthine & 200 & 634 & 539 \\
Oxypurinol & 100 & 67 & 160 \\
\hline
\end{tabular}

$K_{\mathrm{m} / \mathrm{i}}$ values were determined as described in Materials and Methods. n.i., no inhibition ( $90-100 \%$ uptake); $>1000$ indicates $60-75 \%$ inhibition at $1 \mathrm{mM}$. The results are averages of at least three independent experiments with three replicates for each concentration point and the standard deviation was $<20 \%$.

mutations, the second assumption could be dismissed immediately on the basis of the picture from the epifluorescence microscopic analysis shown in Fig. 4d. Thus, N154 should affect the bona fide function of UapA.

To further investigate this idea, we asked whether substitutions S154A and S154N affected the substrate affinity or the specificity of UapA. Table 3 gives a detailed kinetic analysis of S154A and S154N mutants, which establishes that both UapA-S154N and UapA-S154A molecules have substrate affinity profiles different from that of the wild type UapA. In both cases, there is minor two- to three-fold reduction in the affinity for analogs with bulky substitutions at position 8 or 9 of the purine ring (8methylxanthine, 9-methylxanthine, oxypurinol). In addition, S154A confers an $\sim 7$-fold reduction in the affinity for uric acid, whereas S154N leads to moderate increase in the affinity for xanthine and uracil. Thus, Ser154 is important for determining the specificity of UapA.

\section{A UapA model based on the UraA structure}

Molecular simulations were undertaken in order to build a UapA model on the basis of its sequence similarity with the only NAT protein with a known crystallographic structure; namely, the UraA uracil permease of E. coli that was published while this work was in progress. ${ }^{24}$ The UraA structure was captured bound with uracil and corresponds to an inward-facing conformation, occluded from the periplasmic side of the membrane but open towards the cytoplasm. The overall topology of the model UapA shown in Fig. 5 is in very good agreement with the structure of UraA, which consists of 14 TMSs with $\mathrm{N}$ - and C-termini located cytoplasmical- ly (Supplementary Data Table S1). The 14 TMSs are arranged, as in UraA, in two inverted and intermingled repeats (TMS1-TMS7 and TMS8-TMS14) related by a rotation of $\sim 180^{\circ}$. TMS1-TMS4 and TMS8-TMS11 create a core domain, which hosts a putative substrate-binding cavity.

In the model UapA structure, TMS1-TMS7 correspond well to the previously proposed TMSs using different algorithms. The model TMS8 starts 13 amino acid residues downstream from the predicted TMS8. Significant topological discrepancies between the model and predicted structures exist immediately downstream from TMS8. These are concerned mainly with three short interrupted $\alpha$-helices in the model structure (TMS9-TMS10-TMS11), rather than the predicted single long transmembrane segment including helices TMS9a and TMS9b. ${ }^{24,26}$

Consequently, the predicted TMS11-TMS12 corresponds to TMS13-TMS14 in the model structure. It is noticeable that the segment including the last two TMS in UapA is longer than that of UraA. The significance of this observation in relation to the differential functional role of this segment in bacterial and fungal NAT members will be discussed elsewhere (V. Kosti et al., unpublished results). Noticeably, there are two short $\beta$-strands in TMS3 and TMS10 located in the center of the structure, as observed in UraA. The NAT signature motif lies immediately downstream from the $\beta$-strand segment of TMS10 and folds as an $\alpha$-helix. To our satisfaction, the two essential residues proposed to make direct contacts with uracil in UraA (E241 in TMS8 and E290 in TMS10) correspond to E356 and Q408, which we have rigorously shown, by using genetic and biochemical approaches, to be two residues making direct contact with purines in UapA. ${ }^{16,18,19}$

With respect to the domains and residues analyzed in this study, TMS1 and TMS3 are shown to interact with each other and with TMS10, which includes the elements necessary (the NAT signature motif) for substrate binding and transport. The proximity of TMS1 and TMS3 justifies their genetic and functional interaction revealed by the second site-suppressors of H86D. However, His86 is not within bonding distance to Met151, which lies in the side TMS3 that does not face TMS1. This result indicates that suppression of H86D by replacement of Met151 with Ile or Val should occur through an indirect topological modification. This is supported by the observation that M151I or M151V also suppress other H86 mutations (e.g. H86K and H86A), showing the suppression is not allele-specific (data not shown). By contrast, His 86 is within H-bond distance to Asn409 within the signature motif in TMS10, a residue absolutely required for UapA activity. This could very well justify the crucial role of His86 for UapA function. Mutation I74D, which is at the N-terminal border of TMS1, might also somehow affect the crucial interaction of TMS1 
(a)

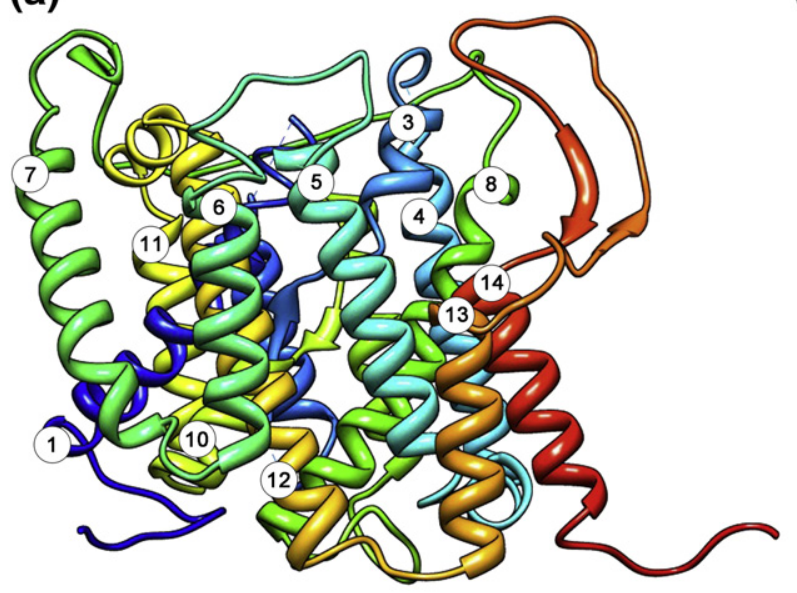

(b)

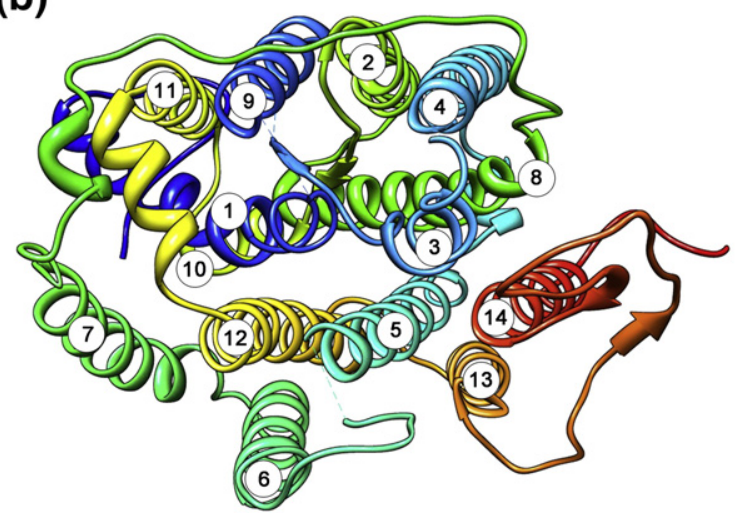

(c)
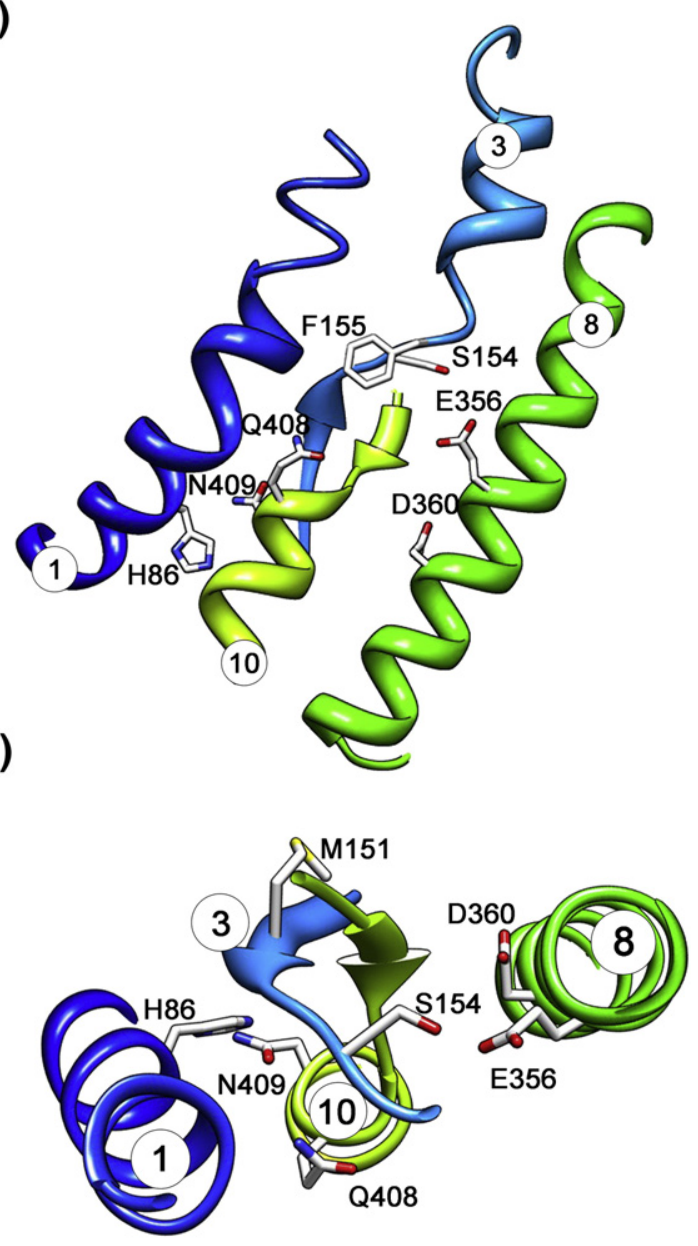

Fig. 5. Model structure of UapA built on the crystal structure of UraA (for details, see the text). The overall structure of UapA as seen from the extracytoplasmic side and from the side are shown in (a) and (b), respectively. Hydrophilic segments 103-117, 137-147, 169-186 and 243-258, which correspond to amino acid sequence alignment gaps with the UraA sequence, could not be structured and are not shown. The numbers of TMSs is indicated. Topological relationships of TMS1, TMS3, TMS8 and TMS10 are shown in more detail in (c) (side view) and (d) (view from the extracytoplasmic side). For clarity, only the parts of the TMS relevant to the functional mutations analyzed here are shown. Amino acid residues genetically and functionally analyzed in this work (His86 in TMS1; Met151, Ser154 and Phe155 in TMS3), and residues involved in substrate binding or transport (Glu356 and Asp360 in TMS8; Gln408 and Asn409 in TMS10) are also shown (see the text). Notice the two antiparallel $\beta$-strands in TMS3 and TMS10 and the close proximity of His86-Asn409 or Ser154-Glu356.

with TMS3 and/or TMS10. Ser154 in TMS3 is in close proximity to Glu356 in TMS8, which might also justify its role in UapA function. Replacement of the other Ser residues in TMS3 with Ala did not produce a significant effect on UapA function, and these Ser residues do not appear to lie in crucial positions in the model UapA structure.

\section{Discussion}

We have presented a functional analysis of rationally designed or genetically selected mutants affecting UapA function and turnover. The relevant mutations analyzed and discussed here are located in regions predicted to correspond to TMS1 and TMS3 in UapA. The concomitant publication of the first NAT crystal structure from E. coli allowed us to build a topological model of UapA and to verify the topology and possible interactions of the mutations discussed in this work. The model UapA structure fully justified our results and interpretations concerning the role of specific residues in TMS1 and TMS3 in UapA folding, turnover and function.

TMS1 appears to be a key domain, as revealed by the pleiotropic character of mutations mapping within it. For example, the absolutely conserved Gln85 residue in the middle of this helix has been 
shown to be essential for transport activity, ${ }^{25}$ whereas the non-conserved Asn71 and Gln113 residues, located in the flanking cytoplasmic and extracellular loops of TMS1 have been shown to be crucial for both UapA specificity and transport kinetics. ${ }^{19}$ None of these purely functionally relevant mutations affect UapA turnover or localization in the plasma membrane. Furthermore, a nonconserved putative Leu zipper motif (Leu77, Leu84, Leu91) has been shown to affect both UapA turnover and transport kinetics, whereas the absolutely conserved His86 and the partially conserved Ile74 were shown, here and earlier, ${ }^{25}$ to affect UapA folding, ER-exit, turnover and specificity. The central position of TMS1 in the UapA model structure, which allows it to make contacts with several other TMS in the core of UapA and, especially, TMS3, TMS8 and TMS10, justifies the genetic and biochemical data obtained with TMS1 mutations.

Ile74, which is predicted to map two amino acid residues upstream from the model TMS1 (Fig. 5b), could be functionally replaced by Ala, Gly or Val, which suggest that what is important in that case is retention of a hydrophobic or a small side chain at position 74. Most other NAT homologs have either hydrophobic (Ile, Leu and Met) or Pro residues at this position (Fig. 4a). This observation suggests that TMS1 in UapA might be longer than that of bacterial homologs and thus include Ile74. In that case, reducing the hydrophobicity of TMS1 through substitutions of Ile74 with polar residues (e.g. I74D and I74N) might affect UapA stability and/ or ERexit. The fact that no other similar case has been met with any hydrophobic to polar replacements in other TMSs suggests that acquisition of proper topology of TMS1 constitutes a crucial element for a functional build-up of UapA. Interestingly, most mutations concerning Ile74 (I74G, I74A and I74V) also reduce the affinity of UapA for uric acid and confer a low but measurable ability to bind other purines. Earlier data from our laboratory have shown that a number of UapA mutant versions or chimeric UapA/UapC molecules have a similarly reduced affinity for uric acid concomitant with increased capacity to recognize other purines. ${ }^{18,19,26}$ On the basis of these observations, we propose that subtle inter-domain interactions specifically evolved in UapA, but not in other known NAT homologs, form the basis for the exceptionally high affinity and high specificity of UapA for uric acid. ${ }^{19,26}$ The results of this study are in accord with this idea and show that TMS1 is a key domain in interdomain interactions.

His86 is one of the few known absolutely conserved residues in NAT carriers. Given that members of the NAT family have different specificities and cation selectivity, we propose that His86 should not be involved directly in substrate or cation recognition. ${ }^{25}$ This prediction is in accord with our present results and the model structure. The genetic analysis showed a functional interaction of His86 (TMS1) with Met151 (TMS3), whereas the model showed a possible physical interaction of His86 (TMS1) with Asn409 (TMS10). Furthermore, Met151 is in the core of the $\beta$-strand of TMS3 which, in the model structure of UapA, appears to be within bonding distance to the $\beta$-strand of TMS10, just upstream from the NAT signature motif, in which Asn409 has been shown to be absolutely essential for UapA-mediated transport, despite the fact that it is not necessary for substrate binding. ${ }^{16,19}$ His 86 and Asn409 substitutions share a similar functional profile, such as cryosensitivity, loss of transport capacity and conservation of substrate-binding ability. ${ }^{16,25}$ A conserved His (TMS1) - Asn (TMS10) interaction in NAT carriers might be crucial for substrate selectivity, as suggested by mutational analysis of the XanQ permease. ${ }^{22}$ In this case, it was shown that specific replacement of the analogous His in TMS1 (e.g. H31Q) recognizes low-affinity novel purine bases and analogs. Some other His31 substitutions (H31C and H31L) have led to impaired XanQ activity whereas others (H31K and H31R) have impaired expression in the plasma membrane, similar to the case of H86A and H86D in UapA. Interestingly, , unlike mutations in His86 in UapA (or His31 in XanQ), those concerning Asn409 (or Asn325 in XanQ) do not affect the topology of the transporter, further suggesting that TMS1 has a more complex role in NAT folding than that of TMS10.

On the whole, our results reveal an important physical and functional interaction of TMS1, TM3 and TMS10 and suggest that mutations in His86 modify the turnover and/or function of UapA through alteration of this interaction. The importance of the interactions of TMS1 with other domains in the NAT family is further supported by work with the human SVCT1 and SVCT2 transporters, which showed recently that the analogous His residue in TMS1 (His51) contributes to substrate binding through a hydrogen bond, whereas mutations in the adjacent conserved Gln residue (Gln50) abolish sodium-dependent ascorbate transport activity. ${ }^{29}$

We showed that Ser154 in TMS3 has a temperature-dependent effect on the activity of UapA. Single Ala replacements of all other Ser residues and of the conserved Phe155, did not produce a significant functional or turnover effect on UapA. Simultaneous replacement of all Ser of TMS3, except Ser154, with Ala residues did not produce a significant defect in UapA function or turnover (Fig. 4). The suggested functional role of Ser154 in UapA is further supported by studies of bacterial NATs permease. ${ }^{22}$ In XanQ, which is a transporter highly specific for xanthine, Asn93 corresponds to Ser154 of UapA. 
Table 4. Forward primers used for gene specific probes, cloning purposes and for mutant construction

\begin{tabular}{|c|c|}
\hline Mutation & Oligonucleotide sequence \\
\hline uapA expBamHI F & 5'-CGGGATCCCTCCATCCATCCATTCAACCGAC-3' \\
\hline uapA XbaI R & 5'-GCTCTAGAGCCTGCTTGCTCTGATACTC-3' \\
\hline hsp70 (ANID_02062.1) F & 5'-CGAGGAGCGCCAGGTCA-3' \\
\hline hsp70 (ANID_02062.1) R & 5'-AACTCCTCGGCCTCGGC-3' \\
\hline 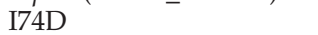 & 5'-GGCCTCAACGAGAAGGATCCCGTGCTGTTGGCGTTTATC-3' \\
\hline $\mathrm{D} 86 \mathrm{H}$ & 5'-GTTTATCCTGGGTCTTCAGCATGCGCTTGCCATGTTGG-3' \\
\hline S145A & 5'-CCAGGTACTATATCGGCGCTGGCGTCCTCTCAGTTATG-3' \\
\hline S149A & 5'-CTATATCGGCAGTGGCGTCCTCGCAGTTATGGGGGTCTCGTTCTC-3' \\
\hline S154A & 5'-GTCCTCTCAGTTATGGGGGTTGCCTTCTCCATCATCTCCGTCGC-3' \\
\hline $\mathrm{S} 154 \mathrm{~N}$ & 5'-GTCCTCTCAGTTATGGGGGTTAACTTCTCCATCATCTCCGTCGC-3' \\
\hline F155A & 5'-CTCAGTTATGGGGGTCTCGGCCTCCATCATCTCCGTCGCC-3' \\
\hline S156A & 5'-GTTATGGGGGTCTCGTTCGCCATCATCTCCGTCGCCAG-3' \\
\hline S159A & 5'-GTCTCGTTCTCCATCATCGCCGTCGCCAGCGGCGCCTTC-3' \\
\hline S162A & 5'-CTCCATCATCTCCGTCGCCGCCGGCGCCTTCAACCAGATG-3' \\
\hline S145A on S149A & 5'-CCAGGTACTATATCGGCGCTGGCGTCCTCGCAGTTATG-3' \\
\hline S159A on S156A & 5'-GTCTCGTTCGCCATCATCGCCGTCGCCAGCGGCGCCTTC-3' \\
\hline S162A on S156/159A & 5'-CGCCATCATCGCCGTCGCCGCCGGCGCCTTCAACCAGATG-3' \\
\hline
\end{tabular}

The nucleobase substitutions of the wild type uapA codon are shown in bold. Reverse primers complementary to the forward primers shown here were used for site-directed mutagenesis.

Replacements N93S and N93A are highly active but more promiscuous for recognition of analogs at the imidazole moiety of the substrate, including uric acid, whereas N93D has low activity. N93T has low affinity for xanthine or analogs and N93Q or N93C is inactive. ${ }^{18}$ It is interesting to note that the N93S replacement in XanQ partly mimics the specificity of fungal (UapA) or plant (Lpe1) ${ }^{30}$ homologs (i.e. recognition of uric acid), which have Ser at this position. Furthermore, in UapA, the mirror image replacement $\mathrm{S} 154 \mathrm{~N}$ increased xanthine recognition (fourfold), whereas S154A has reduced (up to eightfold) uric acid affinity, somehow mimicking the bacterial XanQ permease (Table 3). A similar functional role of the analogous residue in the middle of TMS3 of still another bacterial NAT, the YgfU uric acid permease, was reported recently (K. Papakostas and S. Frillingos, personal communication). Interestingly, Ser154 is close to E356 in TMS8 (Fig. $5 c$ and d), a residue essential for function, 19,22 possibly through its direct interaction with substrates (V. Kosti et al., unpublished results).

\section{Materials and Methods}

\section{Media, strains, growth conditions, and transformation genetics}

Standard media were used for growth of $A$. nidulans and E. coli. ${ }^{31}$ Chemical reagents were obtained from Sigma St. Louis, $\mathrm{MO}$ and from AppliChem $\mathrm{GmbH}$. A strain $\Delta$ uapA $\Delta$ uapC $\triangle a z g A \operatorname{argB2}$ pabaA1 ( $\Delta$ UapA) carrying a singlecopy plasmid integration of the uap $A\left(\mathrm{Uap}^{+}\right)$in the $\arg B$ locus served as a standard wild type control. The parental strain used to select suppressors was $\Delta$ uapA $\Delta$ uapC $\Delta a z g A$ $\arg B 2$ pabaA1 $(\Delta \mathrm{Uap} A)$, expressing the uap $A-\mathrm{H} 86 \mathrm{D}$ or uapA-H86D alleles from a single-copy integrated plasmid.
pabaA1 is a genetic auxotrophy for $p$-aminobenzoic acid. UV mutagenesis is described elsewhere. ${ }^{15}$ An isogenic $\Delta$ uapA $\Delta$ uapC $\Delta a z g A \operatorname{argB2}$ pabaA1 mutant was the recipient strain in transformations, ${ }^{32}$ with uapA alleles constructed or cloned in vectors pAN510GFP and pAN510exp or with an "empty" vector as a negative control (see below). These vectors allow selection of transformants based on arginine auxotrophy complementation. Transformants expressing intact uapA or uapA-gfp alleles, through either single-copy or multi-copy plasmid integration events, were identified by PCR and Southern analysis. In most cases, we selected transformants originating from single-copy homologous integrations in the $\arg B$ locus. Growth tests were performed at $25^{\circ} \mathrm{C}$ and at $37^{\circ} \mathrm{C}, \mathrm{pH}$ 6.8. The standard purine concentration used was $0.5 \mathrm{mM}$ in all earlier studies concerning purine metabolism in $A$. nidulans.

\section{Plasmid constructions and uapA mutations}

pAN510 is a pBluescript vector carrying uapA with its flanking sequences and the $\arg B$ gene as a selection marker. ${ }^{13}$ pAN510-GFP is a pBluescript vector carrying a uap $A-g f p$ fusion with its flanking sequences and the $\arg B$ gene as a selection marker. ${ }^{33}$ pAN510exp is a modified version of pAN510, introducing a BamHI site at the translation start codon and knocking out a XbaI site in the pBluescript multicloning region for cloning purposes. ${ }^{19}$ The uapA orf from the original mutants was amplified by PCR using primers uapA expBamHI F and uapA XbaI R (Table 4), cloned in pAN510exp and sequenced (Macrogen Inc.). Mutations I74D, S145A, S149A, S154A, S154N, F155A, S156A, S159A and S162A alone and in combinations were constructed by site-directed mutagenesis according to the instructions accompanying the QuikChange ${ }^{\circledR}$ Site-Directed Mutagenesis Kit (Stratagene) on vector pAN510-GFP, using complementary oligonucleotides carrying the desired substitution (Table 4), and were confirmed by sequencing. The M151V and M151I mutations were constructed by cloning the H86D/M151V and H86D/M151I open reading frame from the original 
second site H86D suppressors on vector pAN510exp and reversing the first site mutation using primers $\mathrm{D} 86 \mathrm{H}$ (Table 4). The plasmids constructed were introduced by transformation into a $\Delta$ uapA $\Delta u a p C \Delta a z g A \arg B 2$ pabaA1 strain and the resulting integration events were subjected to Southern analysis (see below).

\section{Standard nucleic acid manipulations}

Genomic DNA extraction from A. nidulans was as described. ${ }^{34}$ Plasmid preparation from E. coli strains was done with the Nucleospin Plasmid kit according to the manufacturer's instructions (Macherey-Nagel $\mathrm{GmbH}$ ). DNA bands were purified from agarose gels using the Nucleospin ExtractII kit according to the manufacturer's instructions (Macherey-Nagel $\mathrm{GmbH}$ ). Southern and northern blot analyses were performed as described. ${ }^{35}$ Total RNA extraction from $A$. nidulans was done with the TRIzol ${ }^{\circledR}$ reagent according to the manufacturer's instructions (Invitrogen). In northern blots, equal RNA loading $(5 \sim 10 \mu \mathrm{g})$ was estimated by measurement of absorbance at wavelengths of $260 \mathrm{~nm}$ and $280 \mathrm{~nm}$ and controled by estimating the amount of rRNA as described. ${ }^{35}\left[{ }^{32} \mathrm{P}\right] \mathrm{dCTP}-$ labeled molecules used as $h s p 70$ (ANID_02062.1) or uapAspecific probes were prepared using a random hexanucleotide primer kit following the supplier's instructions (Takara Bio Inc.) and purified on MicroSpin ${ }^{\mathrm{TM}} \mathrm{S}-200 \mathrm{HR}$ columns, following the supplier's instructions (Roche Applied Science). Labeled $\left[{ }^{32} \mathrm{P}\right] \mathrm{dCTP}(3000 \mathrm{Ci} / \mathrm{mmol})$ was purchased from the Institute of Isotopes Co., Ltd. Restriction enzymes were from Takara Bio Inc. Conventional PCR reactions were done with KAPATaq DNA polymerase (KAPABIOSYSTEMS, USA). Cloning and amplification of products were done with Pfx Platinum (Invitrogen) or Phusion ${ }^{\circledR}$ Flash High-Fidelity PCR Master Mix (New England Biolabs).

\section{Membrane protein extraction and western blot analysis}

Cultures for protein extraction were prepared as described. ${ }^{33}$ Mycelia from cultures were harvested, frozen and broken in liquid nitrogen. All subsequent steps were done at $4^{\circ} \mathrm{C}$. Cell extracts were suspended in extraction buffer (50 mM Tris- $\mathrm{HCl} \mathrm{pH} 7.5,150 \mathrm{mM} \mathrm{NaCl}, 5 \mathrm{mM}$ EDTA pH 8.0) supplemented with Protease Inhibitor Cocktail (Sigma) and $0.2 \mathrm{mM}$ phenylmethanesulfonyl fluoride (PMSF). Unbroken cells and debris were removed by centrifugation for $3 \mathrm{~min}$ at $3000 \mathrm{rpm}$. The crude extracts were precipitated with $5 \%(\mathrm{w} / \mathrm{v})$ TCA and centrifuged for $5 \mathrm{~min}$ at $13000 \mathrm{rpm}$. Pellets were recovered and suspended in extraction buffer supplemented with Protease Inhibitor Cocktail and $0.2 \mathrm{mM}$ PMSF. Equal sample loading was estimated by Bradford assay. Total proteins $(50 \mu \mathrm{g})$ were separated by SDS-PAGE $(10 \%(\mathrm{w} / \mathrm{v})$ polyacrylamide gel) and electroblotted (Mini PROTEANTM Tetra Cell, BIORAD) onto a PVDF membrane (Macherey-Nagel GmbH) for immunodetection. The membrane was treated with $2 \%$ (w/v) non-fat dry milk or according to the manufacturer's instructions and immunodetection was done with a primary mouse anti-GFP monoclonal antibody (Roche Applied Science), or a mouse anti-actin monoclonal (C4) antibody (MP Biomedicals) and a secondary goat antimouse IgG HRP-linked antibody (Cell Signaling). Blots were developed by the chemiluminescent method using the West Pico SuperSignal reagent (Pierce) and SuperRX Fuji medical X-Ray films (FujiFILM Europe GmbH).

\section{Kinetic analysis}

$\left[{ }^{3} \mathrm{H}\right]$ Xanthine uptake in conidiospores was assayed at $37^{\circ} \mathrm{C}$ or $25^{\circ} \mathrm{C}$ as described. ${ }^{14,17,36}$ Radiolabeled purines (19.6-33.4 Ci/mmol) were obtained from Moravek Biochemicals (Brea, CA). $K_{\mathrm{i}}$ values were calculated from the Cheng and Prusoff equation:

$$
K_{i}=I C_{50} /[1 /(L / K m)]
$$

where $L$ is the permeant concentration. ${ }^{17}$

\section{Epifluorescence microscopy}

Samples for fluorescence microscopy were prepared as described. $^{33}$ In brief, the samples were incubated on coverslips in liquid Minimal Medium supplemented with urea as nitrogen source for $12-14 \mathrm{~h}$ at $25^{\circ} \mathrm{C}$, observed on an Axioplan Zeiss phase-contrast epifluorescent microscope with appropriate filters, and the resulting images were acquired with a Zeiss MRC5 digital camera using AxioVs40 V4.40.0 software. Images were then processed with Adobe Photoshop CS2 V9.0.2 software.

\section{Model construction, validation and molecular simulations}

Alignment of UapA and UraA was done with ClustalW§ and the best model was built using MODELLER 9.8 software. ${ }^{37}$ A stepwise validation approach was followed, consisting of MD model optimization, comparison with existing experimental data and, finally, visual inspection. First, theoretical models were subjected to 2.5 ns of stochastic dynamics simulation and found to berelatively stable, with a measured RMSD of alpha carbons from starting coordinates not exceeding $3 \AA$. After SD optimization, the best models were tested for structural consistency by considering all available experimental data. Both were in good accordance with mutagenesis and kinetics studies presented earlier and in the present study.

Supplementary materials related to this article can be found online at doi:10.1016/j.jmb.2011.06.024

\section{Acknowledgements}

We thank V. Myrianthopoulos, G. Lambrinides, C. Gournas and V. Taraslia for technical help in modeling, western blots and uptake assays, respectively. V. K. was co-financed by the European Union (European Social Fund - ESF) and Greek national funds through the Operational Program "Education

§www.genome.jp/tools/clustalw 
and Lifelong Learning" of the National Strategic Reference Framework (NSRF) - Research Funding Program: Heracleitus II. Investing in knowledge society through the European Social Fund. S. A and G. D. thank Professor J. Strauss (BOKU University, Vienna) for laboratory facilities and consumables. Work in the laboratory of G.D. is partly supported by the University of Athens (ELKE).

\section{References}

1. Diallinas, G. \& Gournas, C. (2008). Structure-function relationships in the nucleobase-ascorbate transporter (NAT) family: lessons from model microbial genetic systems. Channels (Austin), 2, 363-372.

2. Gournas, C., Papageorgiou, I. \& Diallinas, G. (2008). The nucleobase-ascorbate transporter (NAT) family: genomics, evolution, structure-function relationships and physiological role. Mol. Biosyst. 4, 404-416.

3. Rivas, C. I., Zúñiga, F. A., Salas-Burgos, A., Mardones, L., Ormazabal, V. \& Vera, J. C. (2008). Vitamin C transporters. J. Physiol. Biochem. 64, 357-375.

4. Tsukaguchi, H., Tokui, T., Mackenzie, B., Berger, U. V., Chen, X. Z., Wang, Y. et al. (1999). A family of mammalian $\mathrm{Na}$--dependent L-ascorbic acid transporters. Nature, 399, 70-75.

5. Savini, I., Rossi, A., Pierro, C., Avigliano, L. \& Catani, M. V. (2008). SVCT1 and SVCT2: key proteins for vitamin C uptake. Amino Acids, 34, 347-355.

6. Yamamoto, S., Inoue, K., Murata, T., Kamigaso, S., Yasujima, T., Maeda, J. Y. et al. (2010). Identification and functional characterization of the first nucleobase transporter in mammals: implication in the species difference in the intestinal absorption mechanism of nucleobases and their analogs between higher primates and other mammals. J. Biol. Chem. 285, 6522-6531.

7. Diallinas, G. \& Scazzocchio, C. (1989). A gene coding for the uric acid-xanthine permease of Aspergillus nidulans: inactivational cloning, characterization, and sequence of a cis-acting mutation. Genetics, 122, 341-350.

8. Gorfinkiel, L., Diallinas, G. \& Scazzocchio, C. (1993). Sequence and regulation of the uapA gene encoding a uric acid-xanthine permease in the fungus Aspergillus nidulans. J. Biol. Chem. 268, 23376-23381.

9. Ravagnani, A., Gorfinkiel, L., Langdon, T., Diallinas, G., Adjadj, E., Demais, S. et al. (1997). Subtle hydrophobic interactions between the seventh residue of the zinc finger loop and the first base of an HGATAR sequence determine promoter-specific recognition by the Aspergillus nidulans GATA factor AreA. EMBO J. 16, 3974-3986.

10. Amillis, S., Cecchetto, G., Sophianopoulou, V., Koukaki, M., Scazzocchio, C. \& Diallinas, G. (2004). Transcription of purine transporter genes is activated during the isotropic growth phase of Aspergillus nidulans conidia. Mol. Microbiol. 52, 205-216.

11. Gournas, C., Amillis, S., Vlanti, A. \& Diallinas, G. (2010). Transport-dependent endocytosis and turnover of a uric acid-xanthine permease. Mol. Microbiol. $75,246-260$.
12. Pantazopoulou, A. \& Diallinas, G. (2007). Fungal nucleobase transporters. FEMS Microbiol. Rev. 31, 657-675.

13. Diallinas, G., Valdez, J., Sophianopoulou, V., Rosa, A. \& Scazzocchio, C. (1998). Chimeric purine transporters of Aspergillus nidulans define a domain critical for function and specificity conserved in bacterial, plant and metazoan homologues. EMBO J. 17, 3827-3837.

14. Meintanis, C., Karagouni, A. D. \& Diallinas, G. (2000). Amino acid residues N450 and Q449 are critical for the uptake capacity and specificity of UapA, a prototype of a nucleobase-ascorbate transporter family. Mol. Membr. Biol. 17, 47-57.

15. Amillis, S., Koukaki, M. \& Diallinas, G. (2001). Substitution F569S converts UapA, a specific uric acid-xanthine transporter, into a broad specificity transporter for purine-related solutes. J. Mol. Biol. 313, 765-774.

16. Koukaki, M., Vlanti, A., Goudela, S., Pantazopoulou, A., Gioule, H., Tournaviti, S. \& Diallinas, G. (2005). The nucleobase-ascorbate transporter (NAT) signature motif in UapA defines the function of the purine translocation pathway. J. Mol. Biol. 350, 499-513.

17. Goudela, S., Karatza, P., Koukaki, M., Frillingos, S. \& Diallinas, G. (2005). Comparative substrate recognition by bacterial and fungal purine transporters of the NAT/NCS2 family. Mol. Membr. Biol. 22, 263-275.

18. Vlanti, A., Amillis, S., Koukaki, M. \& Diallinas, G. (2006). A novel-type substrate-selectivity filter and ER-exit determinants in the UapA purine transporter. J. Mol. Biol. 357, 808-819.

19. Papageorgiou, I., Gournas, C., Vlanti, A., Amillis, S., Pantazopoulou, A. \& Diallinas, G. (2008). Specific interdomain synergy in the UapA transporter determines its unique specificity for uric acid among NAT carriers. J. Mol. Biol. 382, 1121-1135.

20. Karatza, P., Panos, P., Georgopoulou, E. \& Frillingos, S. (2006). Cysteine-scanning analysis of the nucleobaseascorbate transporter signature motif in $\mathrm{YgfO}$ permease of Escherichia coli: Gln-324 and Asn-325 are essential, and Ile-329-Val-339 form an alpha-helix. J. Biol. Chem. 281, 39881-39890.

21. Papakostas, K., Georgopoulou, E. \& Frillingos, S. (2008). Cysteine-scanning analysis of putative helix XII in the YgfO xanthine permease: ILE-432 and ASN430 are important. J. Biol. Chem. 283, 13666-13678.

22. Karena, E. \& Frillingos, S. (2009). Role of intramembrane polar residues in the $\mathrm{YgfO}$ xanthine permease: HIS-31 and ASN-93 are crucial for affinity and specificity, and ASP-304 and GLU-272 are irreplaceable. J. Biol. Chem. 284, 24257-24268.

23. Georgopoulou, E., Mermelekas, G., Karena, E. \& Frillingos, S. (2010). Purine substrate recognition by the nucleobase-ascorbate transporter signature motif in the YgfO xanthine permease: ASN-325 binds and ALA323 senses substrate. J. Biol. Chem. 285, 19422-19433.

24. Mermelekas, G., Georgopoulou, E., Kallis, A., Botou, M., Vlantos, V. \& Frillingos, S. (2010). Cysteinescanning analysis of helices TM8, TM9a, and TM9b and intervening loops in the YgfO xanthine permease: a carboxyl group is essential at ASP-276. J. Biol. Chem. 285, 35011-35020.

25. Pantazopoulou, A. \& Diallinas, G. (2006). The first transmembrane segment (TMS1) of UapA contains 
determinants necessary for expression in the plasma membrane and purine transport. Mol. Membr. Biol. 23, 337-348.

26. Kosti, V., Papageorgiou, I. \& Diallinas, G. (2010). Dynamic elements at both cytoplasmically and extracellularly facing sides of the UapA transporter selectively control the accessibility of substrates to their translocation pathway. J. Mol. Biol. 397, 1132-1143.

27. Gregersen, N. \& Bross, P. (2010). Protein misfolding and cellular stress: an overview. Methods Mol. Biol. 648, 3-23.

28. Lu, F., Li, S., Jiang, Y., Jiang, J., Fan, H., Lu, G. et al. (2011). Structure and mechanism of the uracil transporter UraA. Nature, 472, 243-246.

29. Varma, S., Campbell, C. E. \& Kuo, S. M. (2008). Functional role of conserved transmembrane segment 1 residues in human sodium-dependent vitamin $C$ transporters. Biochemistry, 47, 2952-2960.

30. Argyrou, E., Sophianopoulou, V., Schultes, N. \& Diallinas, G. (2001). Functional characterization of a maize purine transporter by expression in Aspergillus nidulans. Plant Cell, 13, 953-964.

31. Cove, D. J. (1966). The induction and repression of nitrate reductase in the fungus Aspergillus nidulans. Biochim. Biophys. Acta, 113, 51-56.
32. Koukaki, M., Giannoutsou, E., Karagouni, A. \& Diallinas, G. (2003). A novel improved method for Aspergillus nidulans transformation. J. Microbiol. Methods, 55, 687-695.

33. Pantazopoulou, A., Lemuh, N. D., Hatzinikolaou, D. G., Drevet, C., Cecchetto, G., Scazzocchio, C. \& Diallinas, G. (2007). Differential physiological and developmental expression of the UapA and AzgA purine transporters in Aspergillus nidulans. Fungal. Genet. Biol. 44, 627-640.

34. Lockington, R. A., Sealy-Lewis, H. M., Scazzocchio, C. \& Davies, R. W. (1985). Cloning and characterization of the ethanol utilization regulon in Aspergillus nidulans. Gene, 33, 137-149.

35. Sambrook, J., Fritsch, E. \& Maniatis, T. (1989). Editors of Molecular Cloning: A Laboratory Manual. Cold Spring Harbor Press, Cold Spring Harbor, NY..

36. Goudela, S., Reichard, U., Amillis, S. \& Diallinas, G. (2008). Characterization and kinetics of the major purine transporters in Aspergillus fumigatus. Fungal Genet. Biol. 45, 459-472.

37. Sali, A., Potterton, L., Yuan, F., van Vlijmen, H. \& Karplus, M. (1995). Evaluation of comparative protein modeling by MODELLER. Proteins: Struct. Funct. Genet. 23, 318-326. 